\title{
The Evolution of the Evolving Neuro-Fuzzy Systems: From Expert Systems to Spiking-, Neurogenetic-, and Quantum Inspired
}

\author{
Nikola Kasabov
}

\begin{abstract}
This chapter follows the development of a class of intelligent information systems called evolving neuro-fuzzy systems (ENFS). ENFS combine the adaptive/evolving learning ability of neural networks and the approximate reasoning and linguistically meaningful explanation features of fuzzy rules. The review includes fuzzy expert systems, fuzzy neuronal networks, evolving connectionist systems, spiking neural networks, neurogenetic systems, and quantum inspired systems, all discussed from the point of few of fuzzy rule interpretation as new knowledge acquired during their adaptive/evolving learning. This review is based on the author's personal (evolving) research, integrating principles from neural networks, fuzzy systems and nature.
\end{abstract}

\subsection{Early work on the integration of neural networks and fuzzy systems for knowledge engineering: Neuro-fuzzy expert systems}

The seminal work by Lotfi Zadeh on fuzzy sets, fuzzy rules and intelligent systems [36-38] opened the field for the creation of new types of expert systems that combined the learning ability of neural networks, at a lower level of information processing, and the reasoning and explanation ability of fuzzy rule-based systems, at the higher level. An exemplar system is shown in Figure 22.1, where at a lower level a neural network (NN) module predicts the level of a stock index and a fuzzy reasoning module combines the predicted values with some macro-economic variables, using the following types of fuzzy rules [18]:

$$
\begin{aligned}
& \text { IF < the predicted by the NN module stock is high }> \\
& \text { AND < the economic situation is good }> \\
& \text { THEN < buy stock }>
\end{aligned}
$$

These fuzzy expert systems continued the development of the hybrid NN-rulebased expert systems that used crisp propositional and fuzzy rules $[13,15,17]$. They represented a major topic at some conferences (Figure 22.2). 


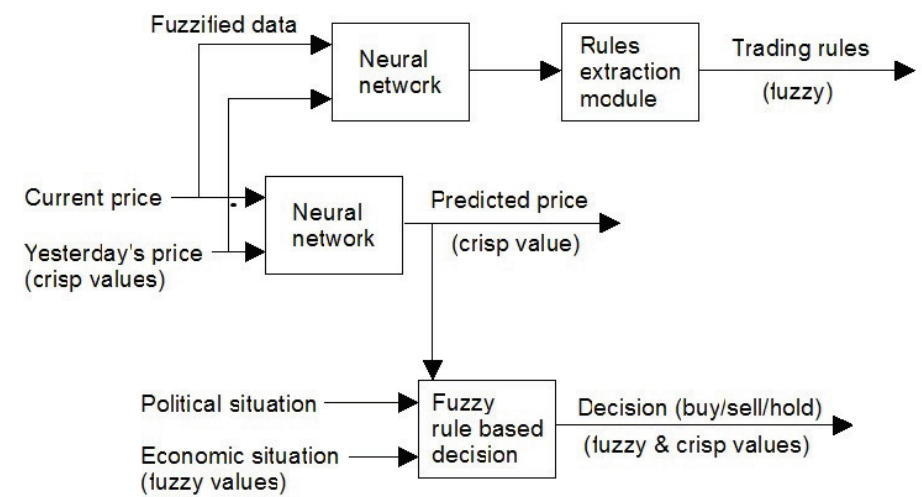

Fig. 22.1. A hybrid NN-fuzzy rule-based expert system for financial decision support (from [18]).

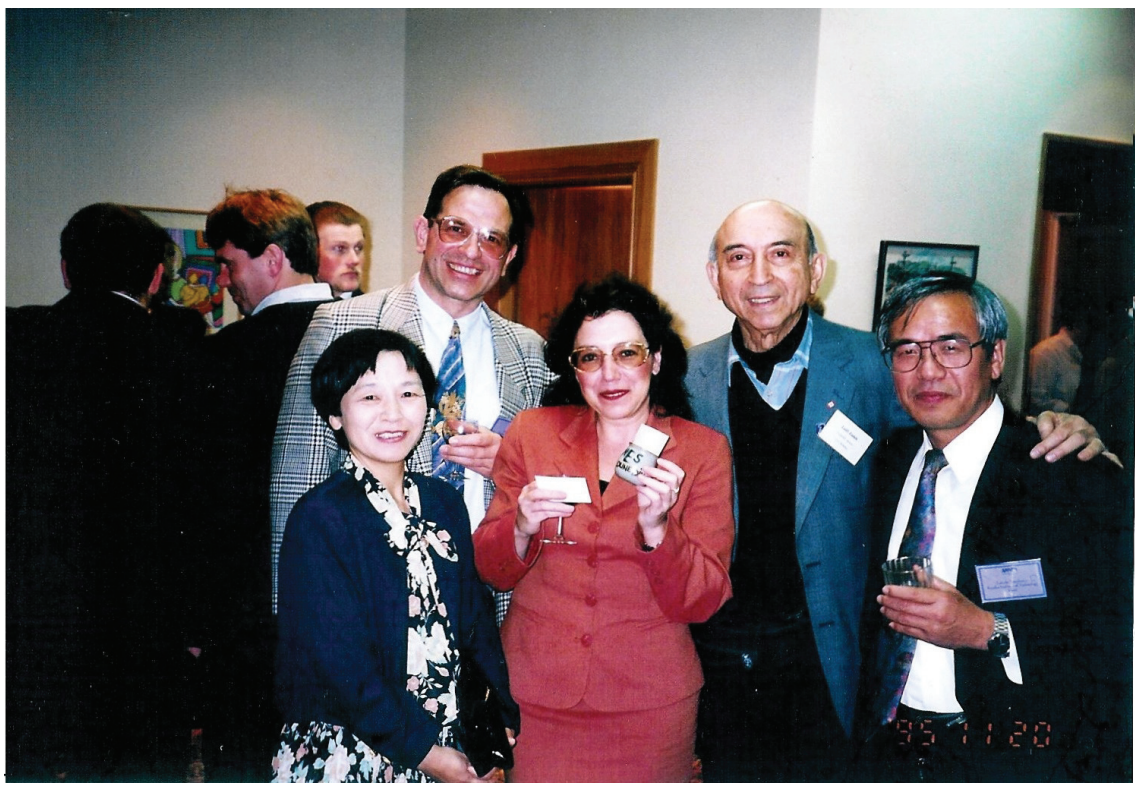

Fig. 22.2. At the 1995 ANNES conference in New Zealand: Lotfi Zadeh with T. Yamakawa, Mrs T. Yamakawa, D. Mehandjiiska-Stavreva and N. Kasabov.

\subsection{Fuzzy neurons and fuzzy neural networks. Evolving connectionist systems}

The low-level integration of fuzzy rules into a single neuron model and larger neural network structures, tightly coupling learning and fuzzy reasoning rules into connectionists structures, was initiated by Prof. Takeshi Yamakawa and other Japanese sci- 
entists and promoted at a series of IIZUKA conferences in Japan [35]. Many models of fuzzy neural networks were developed based on these principles $[6,18,19]$.

The evolving neuro-fuzzy systems developed further these ideas, where instead of training a fixed connectionist structure, the structure and its functionality were evolving from incoming data, often in an on-line, one-pass learning mode. This is the case with the evolving connectionist systems (ECOS) [19-23,31]. ECOS are modular connectionist based systems that evolve their structure and functionality in a continuous, self-organised, on-line, adaptive, interactive way from incoming information [20]. They can process both data and knowledge in a supervised and/or unsupervised way. ECOS learn local models from data through clustering of the data and associating a local output function for each cluster represented in a connectionist structure. They can learn incrementally single data items or chunks of data and also incrementally change their input features $[22,24]$. Elements of ECOS have been proposed as part of the classical NN models, such as SOM, RBF, FuzyARTMap, Growing neural gas, neuro-fuzzy systems, RAN (see [22,24]). Other ECOS models, along with their applications, have been reported in $[7,24,31,32]$.

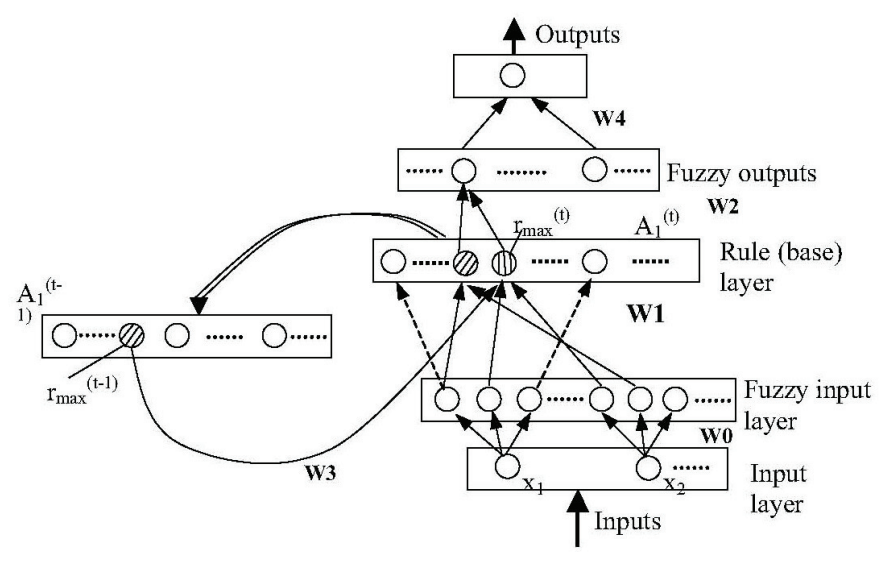

Fig. 22.3. An example of EFuNN model [21].

The principle of ECOS is for neurons to be allocated as centres of fuzzy data clusters and for the system to create local models in these clusters. Fuzzy clustering, as a mean to create local knowledge-based systems, was stimulated by the pioneering work of Bezdek, Yager and Filev [2-4,34]. Here we will briefly illustrate the concepts of ECOS on two implementations: EFuNN [21] and DENFIS [23]. Examples of EFuNN and DENFIS are shown in Figure 22.3 and Figure 22.4 respectively. In ECOS clusters of data are created based on similarity between data samples either in the input space (this is the case in some of the ECoS models, e.g. the dynamic neuro-fuzzy inference system DENFIS), or in both the input and output space (this is the case e.g. in the EFuNN models). Samples that have a distance to an existing 
node (cluster center, rule node) less than a certain threshold are allocated to the same cluster. Samples that do not fit into existing clusters, form new clusters. Cluster centers are continuously adjusted according to new data samples, and new clusters are created incrementally. ECOS learn from data and automatically create or update a local fuzzy model/function, e.g.:

$$
\text { IF < data is in a fuzzy cluster } C_{i}>\text { THEN < the model is } F_{i}>\text {, }
$$

where $F_{i}$ can be a fuzzy value, a linear or regression function (Figure 22.4) or a NN model [22-24].

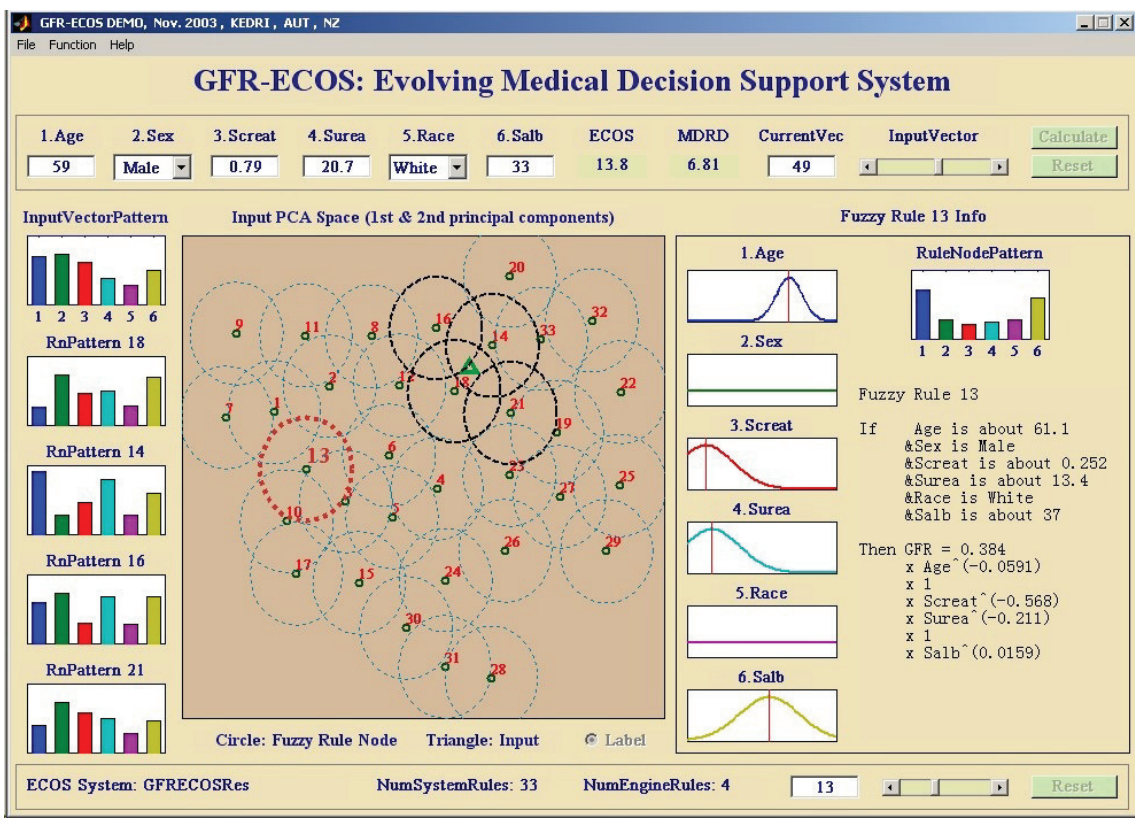

Fig. 22.4. An example of DENFIS model [24] for medical application.

A special direction of ECOS was transductive reasoning and personalised modelling. Instead of building a set of local models (e.g. prototypes) to cover the whole problem space and then use these models to classify/predict any new input vector, in transductive modelling for every new input vector a new model is created based on selected nearest neighbour vectors from the available data. Such ECOS models are NFI and TWNFI [28]. In TWNFI for every new input vector the neighbourhood of closets data vectors is optimised using both the distance between the new vector and the neighbouring ones and the weighted importance of the input variables, so that the error of the model is minimised in the neighbourhood area [25]. 
While the classical ECOS use a simple McCulloch and Pitts model of a neuron, the further developed evolving spiking neural network (eSNN) architectures used a spiking neuron model using the same or similar ECOS principles and applications.

\subsection{Evolving Spiking Neural Networks (eSNN) and Fuzzy Rule Extraction}

A single biological neuron and the associated synapses is a complex information processing machine that involves short term information processing, long term information storage, and evolutionary information stored as genes in the nucleus of the neuron. A spiking neuron model assumes input information represented as trains of spikes over time. When sufficient input information is accumulated in the membrane of the neuron, the neuron's post synaptic potential exceeds a threshold and the neuron emits a spike at its axon (Figure 22.5). Some of the-state-of-the-art models of a spiking neuron include: early models by Hodgkin and Huxley [10], 1952; more recent models by Maas, Gerstner, Kistler, Izhikevich and others, e.g.: Spike Response Models (SRM); Integrate-and-Fire Model (IFM) (Figure 22.5); Izhikevich models; adaptive IFM; probabilistic IFM [11, 12].

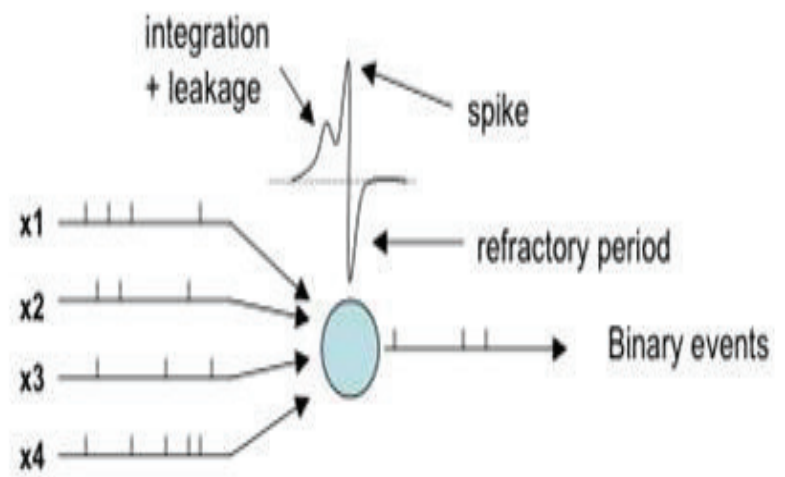

Fig. 22.5. The structure of the LIFM.

Based on the ECOS principles, an evolving spiking neural network architecture (eSNN) was proposed in $[24,33]$ which was initially designed as a visual pattern recognition system. The first eSNNs were based on the Thorpe's neural model [29], in which the importance of early spikes (after the onset of a certain stimulus) is boosted, called rank-order coding and learning. Synaptic plasticity is employed by a fast supervised one-pass learning algorithm. Different eSNN models were developed, including: a reservoir-based eSNN for spatio- and spectro-temporal pattern recognition (Figure 22.6) [30]; eSNN an architecture that used both rank-order and 
time-based learning methods to account for spatio-temporal data [27]; specialised architectures for EEG modelling [24]; moving object recognition systems; etc.

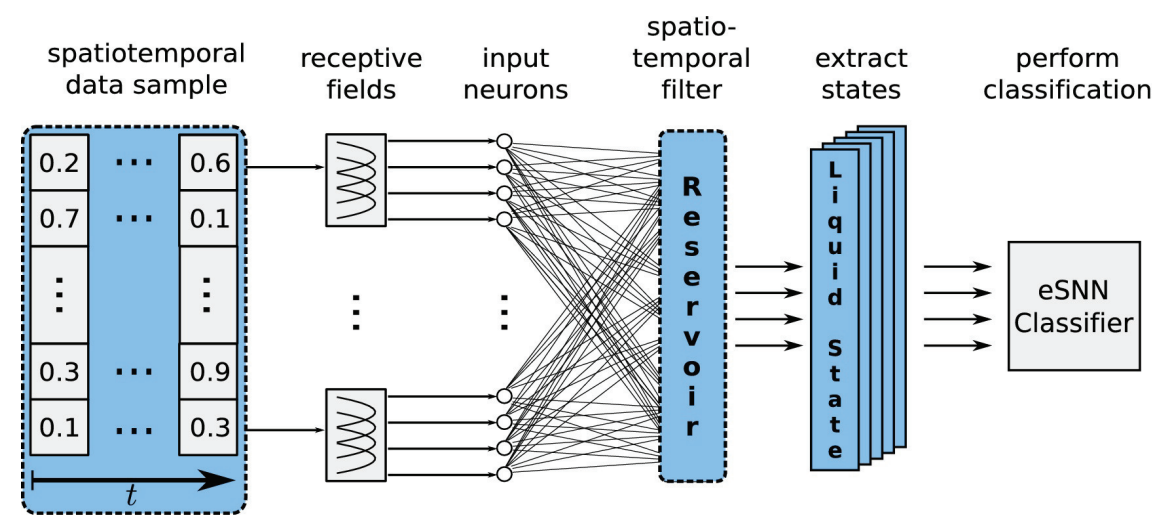

Fig. 22.6. A reservoir-based eSNN for spatio-temporal pattern classification.

Extracting fuzzy rules from an eSNN would make the eSNN not only efficient learning models, but also knowledge-based models. A method was proposed in [26] and illustrated in Figure 22.7). Based on the connection weights $\mathrm{W}$ between the receptive field layer L1 and the class output neuron layer L2 the following fuzzy rules are extracted:

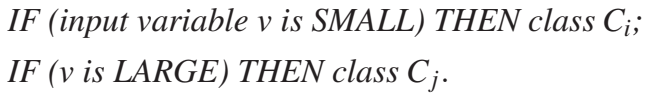

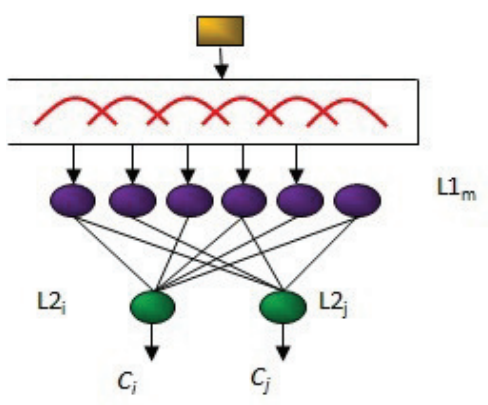

(a)

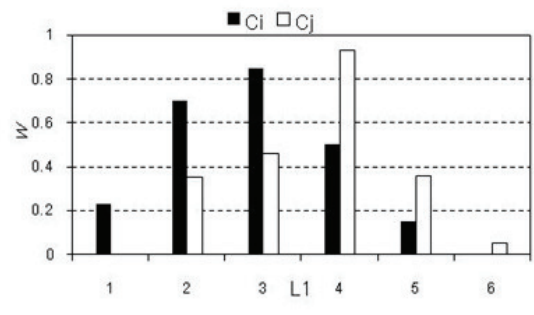

(b)

Fig. 22.7. (a): A simple structure of an eSNN for 2-class classification based on one input variable using 6 receptive fields to convert the input values into spike trains; (b): The connection weights of the connections to class $C_{i}$ and $C_{j}$ output neurons respectively are interpreted as fuzzy rules 


\subsection{Computational Neuro-Genetic Models (CNGM) and Fuzzy Rules}

A neurogenetic model of a neuron is proposed in [24] and studied in [1]. It utilises information about how some proteins and genes affect the spiking activities of a neuron such as fast excitation, fast inhibition, slow excitation, and slow inhibition. An important part of the model is a dynamic gene/protein regulatory network (GRN) model of the dynamic interactions between genes/proteins over time that affect the spiking activity of the neuron - (Figure 22.8).

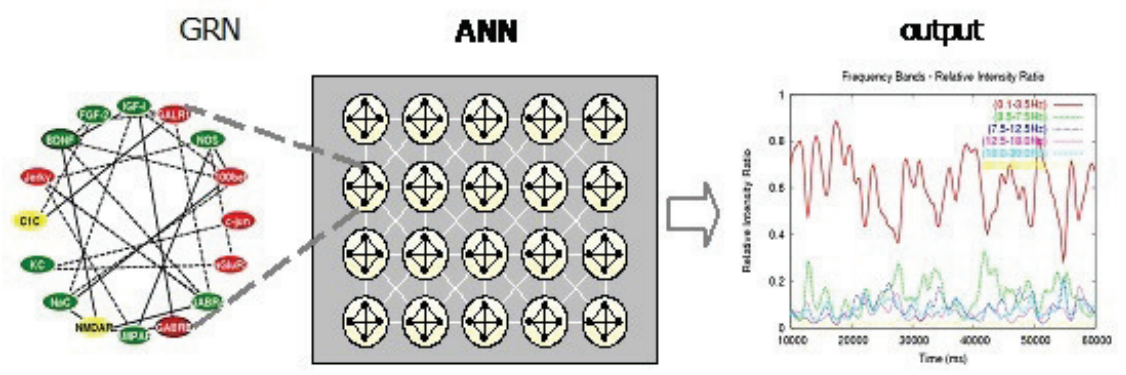

Fig. 22.8. A schematic diagram of a CNGM framework, consisting of a GRN as part of a eSNN [1].

New types of neuro-genetic fuzzy rules can be extracted from such CNGM in the form of:

$$
\begin{aligned}
& \text { IF }<\text { GRN is represented by a function } F> \\
& \text { AND }<\text { input is Small }> \\
& \text { THEN }<\text { Class } C>
\end{aligned}
$$

\subsection{Quantum Inspired SNN (QiSNN)}

QiSNNs use the principle of superposition of states to represent and optimize features (input variables) and gene parameters of the SNN [24]. They are optimized through quantum inspired genetic algorithm [5] or QiPSO. Features or genes are represented as qubits in a superposition of 1 (selected), with a probability $p_{1}$, and 0 (not selected) with a probability $p_{0}$. When the model has to be calculated, the quantum bits 'collapse' in 1 or 0. Fuzzy rules in QiSNN would look like: 
IF $<$ GRN is represented by a function $F$ with a quantum probability $p>$ $A N D<$ input is Small with a quantum probability $q>$

$A N D<$ the model parameters are $S$ with quantum probability $s>$

THEN $<$ Class $C$, with probability $r>$

\subsection{Conclusion}

This chapter presented brief highlights of the development of neuro-fuzzy models for intelligent information systems. The main idea is to facilitate the discovery of new knowledge, along with the development of new connectionist models and systems integrating principles from neural networks, fuzzy systems, evolutionary computation and quantum computing.

\section{Acknowledgment}

The work on this chapter is supported by the Knowledge Engineering and Discovery Research Institute (KEDRI, http://www.kedri.info) and partially by the EU FP7 Marie Curie IIF project EvoSpike (http://ncs.ethz/projects/evospike/. My work on these evolving topics, presented here only as highlights, ideas and principles, have been inspired over the past years by the previous work of pioneers, such as: L. Zadeh, T. Yamakawa, S.-I. Amari, J. Taylor, W. Freeman, M. Arbib, T. Kohonen and many more. I would like to thank Diana Kassabova for helping me with the manuscript and the editors of the volume for their tremendous effort to put together a memorable collection of chapters representing both the history and the state-of-the-art in the field. 


\section{References}

1. Benuskova, Lubica and Nikola Kasabov: Computational Neuro-genetic Modelling, New York: Springer, 2007.

2. Bezdek, James: Pattern Recognition with Fuzzy Objective Function Algorithms, New York: Plenum Press, 1981.

3. Bezdek, James (ed.): Analysis of Fuzzy Information, vols. 1, 2, 3, Boca Raton, Florida: CRC Press, 1987.

4. Bezdek, James: A Review of Probabilistic, Fuzzy, and Neural Models for Pattern Recognition, Journal of Intelligent and Fuzzy Systems, vol. 1, 1993, pp. 1-25.

5. Defoin-Platel, Michael; Schliebs, Stefan and Nikola Kasabov: Quantum-inspired Evolutionary Algorithm: A multi-model EDA, IEEE Transactions on Evolutionary Computation, vol. 13 (6), 2009, pp. 1218-1232.

6. Furuhashi, Takeshi; Hasegawa, Takashi; Horikawa. Shin-ichi and Yoshiki Uchikawa: An Adaptive Fuzzy Controller Using Fuzzy Neural Networks, in: Proceedings of Fifth IFSA World Congress, 1993, pp.769-772.

7. Futschik, Matthias E. and Nikola Kasabov: Fuzzy Clustering in Gene Expression Data Analysis, Proceedings of the World Congress of Computational Intelligence WCCI'2002, Hawaii, May, 2002, IEEE Press, 2002.

8. Gerstner, Wulfram: Time Structure of the Activity of Neural Network Models, Physical Review E, vol. 51, 1995, pp. 738-758.

9. Hebb, Donald: The Organization of Behavior, New York: John Wiley and Sons, 1949.

10. Hodgkin, Allan L. and Andrew F. Huxley: A Quantitative Description of Membrane Current and its Application to Conduction and Excitation in Nerve, Journal of Physiology, vol. 117, 1952, pp. 500-544.

11. Hopfield, John J.: Pattern Recognition Computation Using Action Potential Timing for Stimulus Representation, Nature, vol. 376, 1995, pp. 33-36.

12. Izhikevich, Eugene M.: Which Model to Use for Cortical Spiking Neurons? IEEE Transactions on Neural Networks, vol. 15 (5), 2004, pp. 1063-1070.

13. Kasabov, Nikola: Incorporating Neural Networks into Production Systems and a Practical Approach Towards the Realisation of Fuzzy Expert Systems, Computer Science and Informatics, vol. 21 (2), 1991, pp. 26-34.

14. Kasabov, Nikola: Hybrid Connectionist Production Systems, Journal of Systems Engineering, vol. 3 (1), 1993, pp. 15-21.

15. Kasabov, Nikola and Stephan Shishkov: A Connectionist Production System With Partial Match and its Use for Approximate Reasoning, Connection Science, vol. 5 (3/4), 1993, pp. 275-305.

16. Kasabov, Nikola: Connectionist Fuzzy Production Systems, Springer (LNCS/AI, 847), 1994, pp. 114-128.

17. Kasabov, Nikola: Hybrid Connectionist Fuzzy Production Systems - Towards Building Comprehensive AI, Intelligent Automation and Soft Computing vol. 1 (4), 1995, pp. 351360.

18. Kasabov, Nikola: Foundations of Neural Networks, Fuzzy Systems and Knowledge Engineering, Cambridge, Massachussets, MIT Press 1996.

19. Kasabov, Nikola; Kim, Jaesoo S; Watts Michael and Andrew Gray: FuNN/2 - A Fuzzy Neural Network Architecture for Adaptive Learning and Knowledge Acquisition, Information Sciences - Applications, vol. 101 (3-4), 1997, pp. 155-175.

20. Kasabov, Nikola: Evolving Fuzzy Neural Networks - Algorithms, Applications and Biological Motivation. In: Yamakawa, Takeshi and Gen Matsumoto (eds): Methodologies 
for the Conception, Design and Application of Soft Computing, World Scientific, 1998, pp. 271-274.

21. Kasabov, Nikola: Evolving Fuzzy Neural Networks for on-line Supervised/unsupervised, Knowledge-based Learning, IEEE Transactions on Systems, Man, and Cybernetics, vol. 31 (6), 2001, pp. 902-918.

22. Kasabov, Nikola: Evolving Connectionist Systems: Methods and Applications in Bioinformatics, Brain Study and Intelligent Machines, London, New York, Heidelberg: Springer-Verlag, 2002.

23. Kasabov, Nikola and Qun Song: DENFIS: Dynamic, Evolving Neural-fuzzy Inference Systems and its Application for Time-series Prediction, IEEE Transactions on Fuzzy Systems, vol. 10, 2002, pp. 144-154.

24. Kasabov, Nikola: Evolving Connectionist Systems: The Knowledge Engineering Approach, London: Springer, 2007 (1st edition: 2003).

25. Kasabov, Nikola and Yingjie Hu: Integrated Optimisation Method for Personalised Modelling and Case Study Applications, International Journal of Functional Informatics and Personalised Medicine, vol. 3 (3), 2010, pp. 236-256.

26. Soltic, Snjezana and Nikola Kasabov: Knowledge Extraction From Evolving Spiking Neural Networks With Rank Order Population Coding, International Journal of Neural Systems, vol. 20 (6), 2010, pp. 437-445.

27. Song, Sen.; Miller, Kenneth D. and Larry F. Abbott: Competitive Hebbian Learning Through Spike-timing-dependent Synaptic Plasticity, Nature Neuroscience, vol. 3, 2000, pp. 919-926.

28. Song, Qun and Nikola Kasabov: TWNFI- A Transductive Neuro-fuzzy Inference System With Weighted Data Normalisation for Personalised Modelling, Neural Networks, vol. 19 (10), 2006, pp. 1591-1596.

29. Thorpe, Simon J.; Delorme, Arnaud and Rufin VanRullen: Spike-based Strategies for Rapid Processing, Neural Networks, vol. 14 (6-7), 2001, pp. 715-725.

30. Verstraeten, David; Schrauwen, Benjamin; D'Haene, Michiel and Dirk Stroobandt: An Experimental Unification of Reservoir Computing Methods, Neural Networks, vol. 20 (3), 2007, pp. 391-403.

31. Watts, Michael J.: A Decade of Kasabov's Evolving Connectionist Systems: A Review, IEEE Transactions on Systems, Man and Cybernetics - Part C: Applications and Reviews, vol.39 (3), 2009, pp. 253-269.

32. Widiputra, Harya; Pears, Russel and Nikola Kasabov: Multiple Time-series Prediction Through Multiple Time-series Relationships Profiling and Clustered Recurring Trends. In: Huang, Joshua Zhexue; Cao, Longbing and Jaideep Srivastava (eds.): Advances in Knowledge Discovery and Data Mining - 15th Pacific-Asia Conference, PAKDD 2011 (Proceedings, Part II), Shenzhen, China, May 24-27, 2011, Berlin, Heidelberg: Springer (Lecture Notes in Computer Science 6635, PART 2), 2011, pp. 161-172.

33. Wysoski, Simei Gomes; Benuskova, Lubica and Nikola Kasabov: Evolving Spiking Neural Networks for Audiovisual Information Processing, Neural Networks, vol 23 (7), 2010, pp. pp 819-835.

34. Yager Ronald R. and Dimiter Filev: Generation of Fuzzy Rules by Mountain Clustering, Journal of Intelligent and Fuzzy Systems, vol. 2, 1994, pp. 209-219.

35. Yamakawa, Takeshi; Uchino, Eiji; Miki, Tsutomu and Hiroaki Kusanagi: A Neo Fuzzy Neuron and Its Application to System Identification and Prediction of the System Behaviour, Proceedings of the 2nd International Conference on Fuzzy Logic \& Neural Networks, Iizuka '92, Iizuka, Japan, 1992, pp. 477-483.

36. Zadeh, Lotfi A.: Fuzzy Sets, Information and Control, vol.8, 1965, pp. 338-353. 
37. Zadeh, Lotfi A.: A Theory of Approximate Reasoning. In: Hayes, Jean E. Michie, Donald and L. I. Mikulich (eds.): Machine Intelligence, vol. 9 (based on the International Machine Intelligence Workshop), New York: Elsevier, 1979, pp. 149-194.

38. Zadeh, Lotfi A.: Fuzzy Logic, IEEE Computer, vol. 21, 1988, pp. 83-93. 